\title{
POLÍTICA SOCIAL PARA EL AVANCE DE LA EDUCACIÓN: UNA EVALUACIÓN DEL PROGRAMA DE ESTÍMULOS PREPA SÍ DEL DISTRITO FEDERAL
}

\author{
Mario M. Carrillo-Huerta*
}

(Recibido: octubre 2013/Aceptado: marzo 2014)

\section{Resumen}

Hasta muy recientemente, la educación media superior no había sido obligatoria en México, como no lo es actualmente la educación superior, de manera que la inscripción en esos niveles educativos es baja. ${ }^{1}$ Además, por razones principalmente económicas, la tasa de abandono escolar entre los inscritos es alta. En 2007, el gobierno de la Ciudad de México implantó un programa de estímulos llamado Prepa Sí, orientado a reducir la tasa de deserción escolar y mejorar el desempeño académico de los estudiantes inscritos en escuelas públicas de educación media superior en la ciudad. Este trabajo presenta los resultados de una evaluación del impacto de dicho programa en sus objetivos asignados, realizada con la ayuda de análisis econométrico, aplicado a información proveniente de dos encuestas levantadas entre beneficiarios del programa en el periodo 2008-2009. Se concluye que el programa ha sido exitoso en abatir la tasa de deserción escolar y mejorar el desempeño académico entre sus beneficiarios, y ha tenido impactos positivos adicionales en el bienestar de sus familias.

Palabras clave: política social, educación, programas sociales. Clasificación JEL: I21, I38.

\footnotetext{
* Profesor-investigador del Instituto de Ciencias de Gobierno y Desarrollo Estratégico de la Benemérita Universidad Autónoma de Puebla, y profesor de la Sección de Graduados de la Escuela Superior de Economía del Instituto Politécnico Nacional.

${ }^{1}$ La educación media superior es obligatoria desde el año 2012 en México.
} 


\section{Abstract}

Upper-secondary and higher education in Mexico are not obligatory by law, so that attendance is relatively low, and because mainly of economic reasons, the drop-out rate among those who attend is high. In 2007, the government of Mexico City launched an universal scholarship program called Prepa Sí, aimed at lowering the drop-out rate and improving academic performance among students enrolled in public upper-secondary schools located in that city. This paper presents the results of an evaluation of the impact of that program on its assigned goals, carried out with the help of econometric analysis, applied to data gathered from two surveys directed by the author among beneficiaries of the program in 2009 and 2010. It is concluded that the program is well evaluated by its beneficiaries and has been successful in lowering the drop-out rate and improving academic performance in the city's upper-secondary schools.

Keywords: social policy, education, social programs.

JEL classification: I21, I38.

\section{Introducción}

Aunque México ha logrado una amplia cobertura en educación básica (primaria y secundaria), que ha sido por largo tiempo obligatoria en el país, en el nivel medio superior (y superior) se está muy lejos de alcanzar esos niveles de cobertura, situación que prevalece en todo el país, y por supuesto, también en la Ciudad de México.

De acuerdo con el II Censo General de Población y Vivienda, en 2005, la población de la ciudad con edades entre 15 y 19 años alcanzó la cifra de 740280 habitantes, de los cuales, el 32\% (237 201 jóvenes) no asistían a la escuela, y los que asistían, no mostraban niveles adecuados de desempeño académico. ${ }^{2}$

\footnotetext{
${ }^{2}$ En ese año, la cobertura de la educación en los niveles de primaria y secundaria rebasaron el 95\% en el país. Ese mismo año, en la Ciudad de México habitaban 244884 personas entre 15 y 19 años de edad que habían terminado sus estudios de secundaria, de los cuales solamente 133092 (45.28\%) se inscribieron en el nivel de bachillerato en el siguiente año escolar. De acuerdo con datos de la Encuesta Nacional de la Juventud de 2005, el 31.30\% de la población en el rango de edad de 15-19 años en la ciudad de México, habían abandonado la escuela porque tuvieron que irse a trabajar; es decir, abandonaron por razones económicas. En cuanto al aprovechamiento escolar, en diciembre de 2007, el promedio de calificaciones entre los estudiantes incluidos en Prepa Sí, fue de 7.65 (en una escala del 1 al 10).
} 
En el año 2007, el gobierno de la ciudad implantó un programa de estímulos económicos para la educación pública del nivel medio superior, conocido como Prepa Sí, como parte central de una política social a favor del avance en la educación de su población joven, con dos objetivos principales asignados: inducir a los estudiantes de bachillerato a no abandonar la escuela y a desempeñarse mejor en ella. Desde su implantación, este programa ha sido el más importante en su tipo en la ciudad, representando más del 25\% del presupuesto anual invertido en programas sociales a favor de la educación por el gobierno de la ciudad.

\section{Objetivo general}

El objetivo principal de este trabajo es presentar los resultados de una evaluación de impacto de Prepa Sí en sus objetivos asignados, coordinada por el autor, y realizada con la ayuda de dos modelos econométricos, siguiendo los lineamientos de la metodología de evaluación de programas aplicada regularmente en los casos de los programas de carácter universal. ${ }^{3}$

En las secciones subsecuentes, el trabajo presenta algunas anotaciones de aspectos teóricos tanto en el tema de la política social, como en el de la evaluación de programas sociales. Luego se presenta una descripción general del programa Prepa Sí, seguida de la presentación de los resultados de la evaluación de sus impactos en la retención escolar y en el desempeño académico de los estudiantes, así como en las condiciones de bienestar de los beneficiarios y de sus familiares. Al final del trabajo, se ofrecen algunos comentarios a manera de conclusiones.

\section{Política social y evaluación de programas sociales}

\subsection{Política social}

En términos generales, la política social puede ser definida como “...el diseño y la ejecución programada y estructurada de todas aquellas iniciativas

\footnotetext{
${ }^{3}$ De hecho, el gobierno de la ciudad de México cuenta con un órgano propio de evaluación de programas, conocido como el Consejo de Evaluación del Desarrollo Social del Distrito Federal (EVALUA DF), creado en 2008 con el propósito de establecer la práctica de la evaluación como un elemento regular en la rendición de cuentas. Sin embargo, los esfuerzos de este organismo están orientados primordialmente a emitir los lineamientos para evaluar el diseño, la organización, la cobertura y la operación de los programas, utilizando la metodología de evaluación de la Matriz del Marco Lógico, y por lo regular no incluyen la evaluación de impacto entre sus objetivos primordiales de promoción.
} 
adoptadas para atender una serie de necesidades consideradas básicas para la población..." (Donati, 1985; Montoro, 1997). Como tal, la política social existe en todo el mundo. Sin embargo, se reconoce que su importancia es mayor en regiones en desarrollo, donde las necesidades insatisfechas de bienes y servicios, públicos y privados son mayores en términos relativos al tamaño de la población y a los niveles de bienestar.

Asociada siempre con la intervención del Estado (y gobierno), se considera que la política social incluye tres áreas de intervención directa: 1) la regulación de la economía de mercado para promover la existencia de altas tasas de empleo estable; 2) el reconocimiento de la responsabilidad de asegurar la provisión de un conjunto de servicios sociales, tales como la educación, el ingreso, la salud, la vivienda y otros servicios personales, necesarios para tener niveles razonables de bienestar, en una escala universal, $y, 3$ ) asegurar la existencia de una red amplia de seguridad social basada en servicios asistenciales, para resolver casos de extrema pobreza y abandono entre la población (Mishra, 1990).

Sin embargo, el grado de intervención del Estado en asuntos sociales a través de política social, así como el modelo utilizado para la intervención, dependen de la orientación ideológica de la sociedad. Una orientación liberal aboga por una menor intervención estatal, contraria a una orientación socialdemócrata, que prefiere una amplia intervención directa para proporcionar un nivel mínimo de bienestar a todos los miembros de la sociedad.

Montoro (1997) ha clasificado los modelos que los gobiernos con orientaciones diferentes han adoptado para la implantación de la política social en dos grupos principales, dependiendo del fin último asignado a la política, así como de la manera en que dicha política es implantada. ${ }^{4}$

De acuerdo con su último fin, la política social puede ser un instrumento para: $a$ ) ayudar a las gentes muy pobres o marginadas de la vida social a través de la caridad, sobre simples consideraciones éticas; $b$ ) conseguir cierto control social, para mantener cierta integración social, paz y orden público, $y,{ }^{5} c$ ) cumplir

\footnotetext{
${ }^{4}$ De hecho, dicho autor considera tres tipos de modelos, aunque insiste en que el tercero de ellos no es muy común. De acuerdo con este tercer tipo de modelos, la política social puede ajustarse a: $a$ ) el modelo formal, donde los programas sociales se implantan sin ningún interés en sus impactos o resultados; $b$ ) el modelo normativo, según el cual los programas son evaluados en términos de las discrepancias entre su diseño y sus resultados, $y, c$ ) el modelo materialista, que pone énfasis en sus resultados, sobre la base del reconocimiento de clases sociales y consideraciones de poder.

${ }^{5}$ En este caso, la política social es aplicada por una amplia gama de gobiernos con diferente orientación ideológica, sobre una base pragmática y sin ningún tipo de consideraciones éticas, lo que puede interpretarse como un medio para estabilizar la relación entre los sectores productivos y los no-productivos de la población, para asegurar la estabilidad del sistema social.
} 
con la responsabilidad de satisfacer derechos sociales reconocidos, y por tanto la obligación de atender a todos los miembros de la sociedad. ${ }^{6}$

Dependiendo de la manera en que es implantada, la política social puede ajustarse a: a) un modelo residual, que supone la existencia de dos vías a través de las cuales se canalizan las necesidades sociales: la familia y el mercado, de manera que la política social debería aplicarse sólo cuando alguno de esos dos canales fallara, y debería por tanto tener carácter temporal; ${ }^{7}$ b) el modelo ejecutivo, basado en teorías económicas y sicológicas que reconocen la eficiencia de los incentivos y recompensas, y el cual propone que la ayuda solamente se debe dar en función del mérito individual y de la capacidad productiva (ejecutiva), y, c) el modelo institucional-redistributivo, que considera al bienestar social como un valor supremo, y por tanto propone la intervención directa de las instituciones sociales para ayudar a la gente en función de sus necesidades, como un intento de promover la igualdad social.

\subsection{La evaluación de programas sociales}

La evaluación de los programas sociales oficiales es necesaria generalmente para efectos de rendición de cuentas en cuanto al uso adecuado de recursos públicos. Específicamente, la evaluación ayuda a darles seguimiento al diseño, implantación y operación de los programas, para tener una retroalimentación que permita el mejoramiento en su desempeño. De hecho, la evaluación de los impactos de un programa social, les permite a sus responsables decidir si el programa fue una decisión adecuada para alcanzar los objetivos asignados, para entonces decidir si el programa debería continuar operando o debería abandonarse.

De acuerdo con Baker (2000), comúnmente se tienen algunas preguntas relacionadas con un programa social, tales como: ¿La intervención del Estado está produciendo los beneficios esperados? ¿El programa fue diseñado de la mejor manera posible para alcanzar los resultados esperados? ¿Se están usando eficientemente los recursos públicos asignados

\footnotetext{
${ }^{6}$ En este modelo, la política social “...opera dentro del amplio marco legal y político, distante de consideraciones ideológicas, involucrando por lo tanto a todas las fuerzas y los agentes sociales..." (Montoro, 1997: 43-44).

${ }^{7}$ Desde este punto de vista, el objetivo real de la política social es “...enseñar a la gente a resolver sus propios problemas, en vez de resolvérselos con la acción del Estado..." ((Montoro, 1997; 44).
} 
al programa? ¿Cuáles son los efectos del programa en la población? Esta autora considera que tales preguntas pueden ser contestadas sólo a través de la evaluación de los impactos de los programas, aislándolos de otros factores posibles. ${ }^{8}$

La evaluación de los impactos de los programas sociales siempre involucra a una comparación de dos condiciones diferentes. La comparación más simple es la que se hace entre las condiciones existentes antes y después de la intervención de los programas dentro de algún grupo de individuos claramente definidos. Sin embargo, en la mayoría de los casos, los programas intentan llegar a sólo algunas partes de la población, de modo que las comparaciones tienen que hacerse ente dos grupos de individuos: uno que forma parte del programa, y otro que no lo hace, pero que pudo haberlo hecho. Las dificultades metodológicas en este último caso son considerables, principalmente debido a que es realmente difícil encontrar a los individuos que pudieron ser parte del programa pero no lo son (Heckman et al., 1998).

De hecho, la mayoría de los avances en la metodología de la evaluación de impactos se han orientado a resolver ese problema, principalmente construyendo lo que ha sido llamado el grupo contrafactual; es decir, un grupo imaginario de individuos que pueden ser semejantes a los participantes del programa en todos los aspectos, excepto en el de participar en él. ${ }^{9}$

No obstante, cuando los programas sociales incluyen a todos los miembros de su población objetivo, no existe ni la necesidad de comparar a grupos diferentes, ni la de construir un grupo contrafactual. En esos casos (como lo es el del programa Prepa Sî), solo es necesario comparar las condiciones existentes antes y después de la implantación del programa entre sus beneficiarios. Sin embargo, aún en esos casos prevalece la pregunta de

\footnotetext{
${ }^{8}$ La evaluación de impactos de los programas sociales es especialmente importante para las zonas en desarrollo, donde los recursos son tan escasos que la gente espera que cada peso invertido en ellos debe tener el máximo efecto posible en la reducción de la pobreza. Si los programas sociales no están bien diseñados y no producen los resultados esperados, entonces la información relevante de la evaluación puede ser usada para su rediseño y mejoramiento o incluso para eliminarlos si fuera necesario (Hernández, 2004).

${ }^{9}$ La determinación de un grupo contrafactual es esencial para tener un proceso de evaluación correctamente diseñado. Los enfoques que pueden adoptarse para tal efecto pueden ser de dos tipos: el diseño experimental (aleatorio) y el cuasiexperimental. También pueden ser usados métodos cualitativos y cuantitativos para el diseño. Pero independientemente del enfoque y del método utilizado, los problemas para detectar los efectos atribuibles a un programa siguen siendo considerables (Heckman, 2001; Baker, 2000).
} 
si los impactos en sus objetivos asignados se deben realmente a la intervención del programa, o son el resultado de la presencia de otros factores. Pero ese problema puede resolverse razonablemente bien con el uso de métodos cuantitativos tradicionales aplicados a datos duros, tales como el análisis de regresión, o a través de encuestas de opinión entre los participantes del programa. $^{10}$

\section{Características generales de Prepa Sí}

El objetivo general del programa Prepa Sí es asegurar que todos los estudiantes inscritos en escuelas públicas del nivel medio superior en la Ciudad de México, puedan seguir realizando sus estudios con éxito, sin tener que abandonarlos por razones económicas. Los objetivos específicos del programa incluyen los siguientes: $a$ ) evitar que los estudiantes del nivel escolar medio superior de la ciudad abandonen sus estudios por razones económicas; $b$ ) ayudar a los estudiantes a mejorar su desempeño académi$\mathrm{co}, \mathrm{y}, \mathrm{c}$ ) ayudar a mejorar las condiciones de vida de los estudiantes.

El programa ofrece estímulos económicos a todos los residentes de la Ciudad de México inscritos en escuelas públicas del nivel medio superior. El estímulo es continuo durante 10 meses (equivalentes a un año lectivo) prorrogable anualmente. El monto del estímulo depende del desempeño académico de los estudiantes. Los estudiantes con un promedio de calificaciones entre 6.0 y 7.5 (en una escala del 1 al 10), reciben 500 pesos por mes; aquéllos con promedios entre 7.6 y 9.0, reciben 600 y, quienes tienen promedios entre 9.0 y 10.0, reciben 700 cada mes. Los estímulos son depositados en cuentas bancarias especiales que el programa les abre a los beneficiarios, quienes reciben una tarjeta que les permite checar sus balances o retirar fondos. Los estudiantes también reciben un bono de 1000 pesos por año dentro del programa, entregables al término de los estudios de bachillerato. ${ }^{11}$

\footnotetext{
${ }^{10}$ Como podrá verse en una sección posterior (sección 5), esos dos enfoques fueron usados para evaluar los impactos de Prepa Sí en sus objetivos asignados, tomando como base la información obtenida a partir de dos encuestas, coordinadas por el autor, levantadas entre los beneficiarios del programa.

${ }^{11}$ Sin embargo, para motivar e incentivar el desempeño académico de los estudiantes, el programa les ofrece beneficios adicionales, que incluyen un seguro contra accidentes escolares, un seguro de vida, y acceso preferencial a los eventos que ofrecen las diferentes dependencias del gobierno de la Ciudad.
} 


\subsection{Actividades en comunidad}

Aparte de que los beneficiarios deben estar inscritos oficialmente en una escuela pública de educación media-superior en la ciudad como requisito para ser aceptado en el programa, los estudiantes tienen que participar en actividades en comunidad para permanecer en él. Tal condición es impuesta como la contribución que la Ciudad recibe de los beneficiarios, como respuesta al estímulo entregado, y también para que los estudiantes sientan que de alguna manera se están ganando ese recurso, y que no es un simple regalo de la sociedad. Esas actividades pueden realizarse ya sea en las instalaciones donde estudian los beneficiarios, o en lugares definidos por las autoridades del Programa.

Las actividades en comunidad son multidisciplinarias y agrupadas en cuatro categorías diferentes. Esas categorías y sus objetivos se enlistan a continuación:

Actividades educativas, culturales y de innovación. Consisten en el desarrollo de programas de lectura para la población de adultos en las zonas pobres de la ciudad; la organización de talleres y cursos de regularización para estudiantes de educación media superior; la promoción de la innovación tecnológica, y, la organización de concursos académicos y eventos culturales (música, danza, artes plásticas, entre otros).

Actividades de protección ambiental. Son desarrolladas para promover la protección ambiental, con base en el Plan Verde del gobierno de la Ciudad de México. Esas actividades incluyen las siguientes campañas para el uso adecuado del agua: regeneración, rescate y mejoramiento de espacios públicos y áreas verdes; campañas de promoción de movilidad no motorizada, y, promover separación y reciclaje de la basura.

Actividades y eventos deportivos. Aquí, el objetivo es promover actividades recreativas y deportivas, así como actividades físicas en las comunidades donde habitan los beneficiarios.

Actividades de apoyo general a la comunidad. Estas actividades son promovidas para mantener al día a los miembros de la comunidad, con información de los programas que el gobierno local ofrece a la población de la ciudad, en las áreas de educación; desarrollo de la niñez y de la juventud; protección ambiental; seguridad pública y transporte; vida cultural; deportes y recreación; protección de la salud, y, participación ciudadana en actividades públicas. 


\subsection{Evolución del programa}

El programa arrancó oficialmente el 15 de agosto de 2007. Desde su implantación, Prepa Sí ha sido el programa social a favor de la educación más importante del gobierno de la ciudad. El cuadro 1 y la figura 1, muestran que, desde el año 2008, Prepa Sí ha representado por lo menos el 25\% del presupuesto total de la ciudad, asignado a los programas sociales a favor de la educación. ${ }^{12}$ Por otro lado, la figura 2 muestra que el número de beneficiarios incluidos en el programa durante el periodo 2007-2012 ha promediado alrededor de 200000 estudiantes por año escolar.

\section{Cuadro 1}

Presupuesto del gobierno de la Ciudad de México, asignado a programas sociales a favor de la educación (millones de pesos a precios corrientes)

\begin{tabular}{|ccccc|}
\hline Año & $\begin{array}{c}\text { Prepa Sí } \\
\text { (a) }\end{array}$ & $\begin{array}{c}\text { Otros programas } \\
\text { a favor de la } \\
\text { educación } \\
\mathbf{( b )}\end{array}$ & $\begin{array}{c}\text { Total } \\
(\mathbf{c})=(\mathbf{a}+\mathbf{b})\end{array}$ & $\begin{array}{c}\text { Presupuesto } \\
\text { relativo } \\
(\mathbf{a} / \mathbf{c})\end{array}$ \\
\hline 2001 & 0 & 347.1 & 347.1 & 0 \\
2002 & 0 & 411.9 & 411.9 & 0 \\
2003 & 0 & 744.6 & 744.6 & 0 \\
2004 & 0 & 1417.1 & 1417.1 & 0 \\
2005 & 0 & 1398.2 & 1398.2 & 0 \\
2006 & 0 & 1591.1 & 1591.1 & 0 \\
2007 & 336.1 & 1807.3 & 2243.1 & 0.15 \\
2008 & 1137.3 & 2635.6 & 3772.9 & 0.34 \\
2009 & 1186.8 & 2881.7 & 4068.5 & 0.29 \\
2010 & 1128.5 & 2999.2 & 4127.7 & 0.27 \\
2011 & 1160.3 & 3244.6 & 4404.9 & 0.26 \\
2012 & 1096.8 & 3462.4 & 4529.2 & 0.25 \\
\hline
\end{tabular}

Fuente: reportes de presupuestos de la Secretaría de Finanzas del GDF, 2001-2012.

\footnotetext{
${ }^{12}$ Los datos del cuadro 1 sólo intentan mostrar la importancia relativa de Prepa Sí ante otros programas semejantes.
} 


\section{Figura 1}

Presupuesto del gobierno de la Ciudad de México, asignado

a programas sociales a favor de la educación

(millones de pesos a precios corrientes)

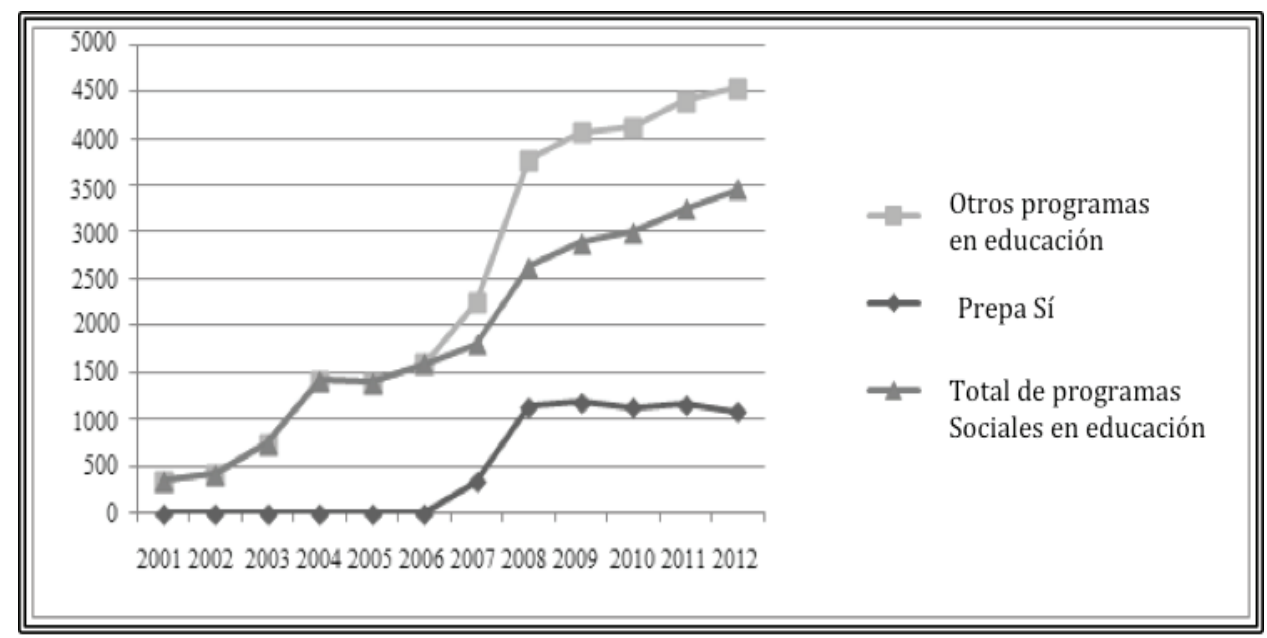

Fuente: cálculado con datos del cuadro 1.

Como ya se ha dicho, Prepa Sí se implantó al inicio del ciclo escolar 2007-2008 (agosto de 2007), y por ser un programa nuevo (y por lo tanto no muy conocido entre la población estudiantil), solamente incorporó alrededor de 130000 estudiantes en diciembre de 2007, no obstante que su población objetivo eran 200000 de ellos. Sin embargo, de acuerdo con la dependencia responsable del programa (el Fideicomiso Educación Garantizada), una vez que Prepa Sí fue del conocimiento generalizado entre los estudiantes, al Programa se incorporó un número considerable de beneficiarios (casi 230 000) durante el ciclo escolar 2008-2009 (figura 2).

\section{Resultados de la evaluación de los impactos de Prepa Sí}

La evaluación de los impactos de Prepa Sí intentó dimensionar los efectos del programa en sus objetivos asignados y consistió en dos ejercicios. En el primero de ellos se calcularon la tasa de deserción y el promedio de calificaciones entre los beneficiarios, a partir de información estadística proporcionada por la instancia responsable de la operación del programa. En este 
ejercicio también se detectaron los impactos del programa en los ingresos familiares y en la satisfacción de los beneficiarios y sus familiares, a partir de la información obtenida de dos encuestas levantadas entre ellos du rante el ciclo escolar 2008-2009.13

\section{Figura 2}

Evolución de la población de beneficiarios del programa Prepa Sí, por ciclo escolar 2007-2012

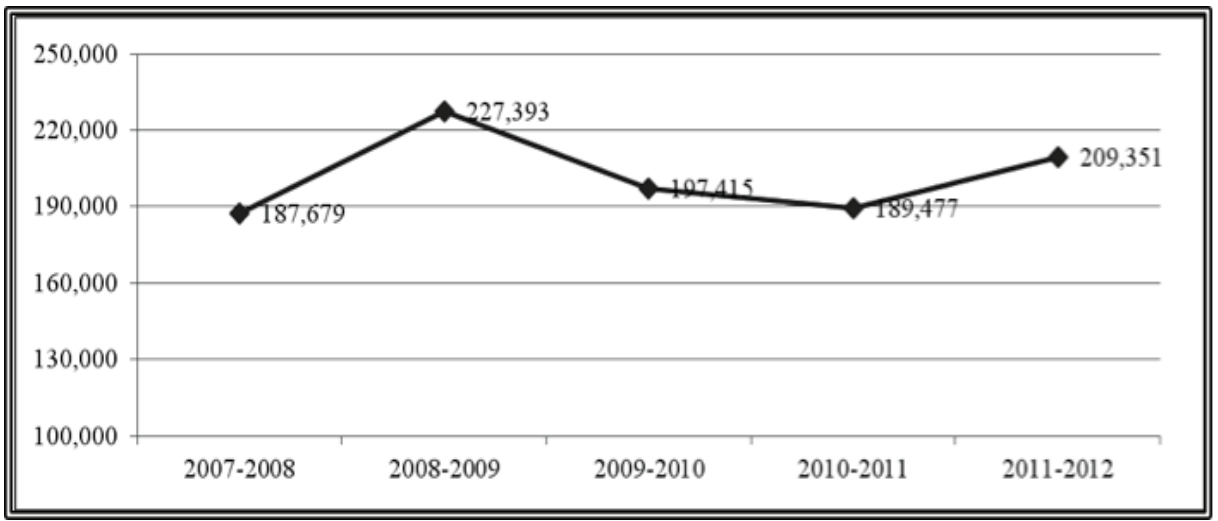

Fuente: Dirección de Evaluación del Fideicomiso Educación Garantizada con información de la Coordinación Ejecutiva del PREBU (2012).

El segundo ejercicio consistió en la aplicación de dos modelos econométricos Probit, aplicados a la información proveniente de las encuestas, con el fin de inferir los impactos del programa, tanto en el desempeño académico como en la decisión de continuar en la escuela, por parte de los beneficiarios.

${ }^{13}$ El tamaño de la muestra para la primera encuesta fue de 1836 beneficiarios de una población total de 196345 estudiantes. El tamaño de la segunda muestra fue de 1980, de una población total de 210044 beneficiarios. En ambos casos, el tamaño de la muestra fue calculado mediante la fórmula: $n=\frac{n_{0}}{1+n_{0} / N}=\frac{n_{0}}{1+\frac{n_{0}}{N}}$, donde $n$, es el tamaño de la muestra, y $N$, el tamaño de la población. Se decidió que el error fuera equivalente al $10 \%$, de manera que: $\operatorname{Pr}\left(\frac{|N p-N P|}{N P} \geq 0.1\right)=\operatorname{Pr}(|p-P \geq r P|)=a$. También se decidió que el valor máximo posible para $p q$ fuera de: $p q=0.25$, y el nivel de confianza fuera del 95\%, de manera que: $\alpha=0.05, \mathrm{y} z=1.96$. 


\subsection{Tasa de deserción, desempeño académico y bienestar de los beneficiarios}

En el ciclo escolar 2006-2007, la tasa de deserción en el nivel medio superior en la Ciudad de México fue de alrededor de $16 \%$, y un año después de la implantación del Programa Prepa Sí, durante el ciclo escolar 2007-2008, la tasa de deserción entre sus beneficiarios disminuyó al 6.08\%. La gráfica 1 muestra la evolución de la tasa de deserción del nivel medio superior en la Ciudad durante el periodo 2000-2010.

En cuanto al desempeño académico de los estudiantes, el promedio de calificaciones entre los beneficiarios del programa aumentó de 7.65 en diciembre de 2007 a 8.39 en junio de 2008. En ese año, el porcentaje de estudiantes dentro del rango de calificaciones de 6.0 a 7.5 bajó de $46 \%$ a $43 \%$, mientras que el correspondiente al rango 7.6 - 9.0 aumentó de $44 \%$ a $46 \%$, y el correspondiente al rango entre 9.1 y 10.0, aumentó del 10\% al 11\%. Y aunque el impacto reciente del programa en el promedio de calificaciones no ha sido sustantivo, éste ha sido positivo. En el cuadro 2, se muestra la evolución del promedio asociada con el programa, entre los ciclos escolares 2007-2008 y 2011-2012.

En cuanto al efecto en el bienestar familiar, una encuesta realizada por la instancia responsable del programa en junio de 2012, entre los beneficiarios y sus familias, mostró que el estímulo económico tiene un impacto muy alto en los hogares de ingresos bajos. En el caso de los hogares de los beneficiarios con un ingreso equivalente a un salario mínimo o menor (el 5.2\% del total de hogares), el estímulo económico del programa representa un $44 \%$ del ingreso total familiar, mientras que para aquellos con un ingreso equivalente a más de un salario mínimo y hasta dos, el estímulo representó el 32\% del ingreso familiar. Es decir, el estímulo del programa permite cubrir costos relacionados con la educación, pero también contribuye al ingreso familiar, reduciendo así la presión de tener que trabajar. 


\section{Gráfica 1}

Tasa de deserción en el nivel mediosuperior de educación en la Ciudad de México, por ciclo escolar, 2000-2010

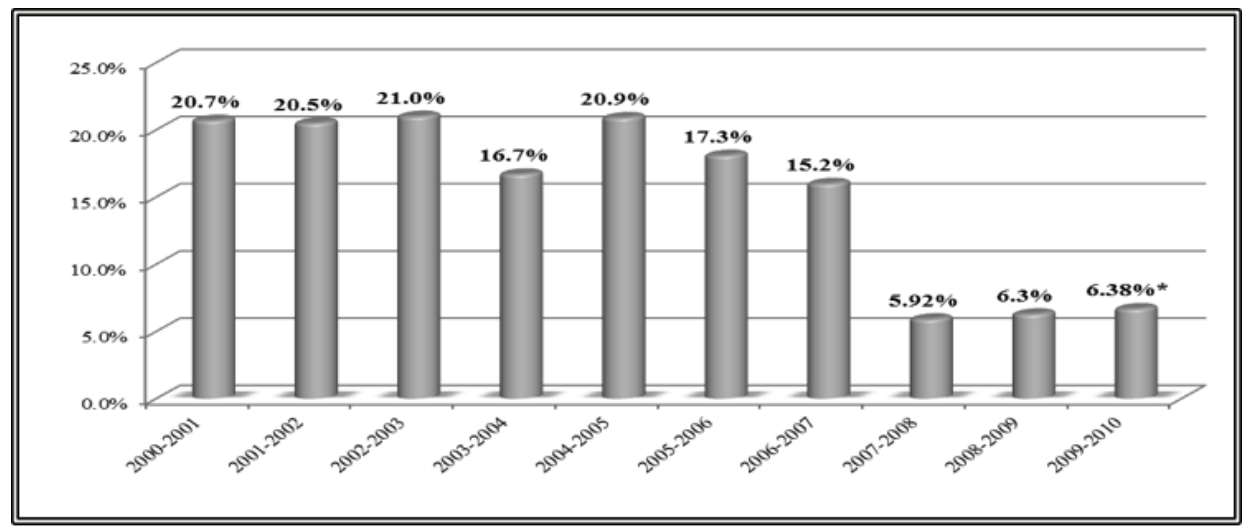

Fuente: SEP, Sistema Educativo de los Estados Unidos Mexicanos, para los ciclos escolares del 2000-2001 al 2007-2008. De 2007 en adelante, la tasa de deserción fue calculada solo para la población de beneficiarios de Prepa Sí.

\section{Cuadro 2}

Evolución del promedio de calificaciones de los estudiantes dentro del programa Prepa Sí, por año de inscripción y ciclo escolar, entre 2007-2008 y 2011-2012

\begin{tabular}{|c|c|c|c|c|c|c|c|c|c|}
\hline \multirow{2}{*}{$\begin{array}{c}\text { Generación } \\
\text { estudiantil } \\
\text { del } \\
\text { programa }\end{array}$} & \multicolumn{2}{|c|}{$\begin{array}{l}\text { Ciclo escolar } \\
\text { 2007-2008 }\end{array}$} & \multicolumn{2}{|c|}{$\begin{array}{l}\text { Ciclo escolar } \\
\text { 2008-2009 }\end{array}$} & \multicolumn{2}{|c|}{$\begin{array}{c}\text { Ciclo escolar } \\
2009-2010\end{array}$} & \multicolumn{2}{|c|}{$\begin{array}{c}\text { Ciclo escolar } \\
\text { 2010-2011 }\end{array}$} & \multirow{2}{*}{$\begin{array}{c}\begin{array}{c}\text { Ciclo } \\
\text { escolar }\end{array} \\
2011-2012 \\
\text { Inicial }\end{array}$} \\
\hline & Inicial & Final & Inicial & Final & Inicial & Final & Inicial & Final & \\
\hline $\begin{array}{c}\text { primera } \\
\text { generación }\end{array}$ & 7.65 & 8.39 & 8.39 & 8.41 & 8.41 & 8.49 & & & \\
\hline $\begin{array}{l}\text { segunda } \\
\text { generación }\end{array}$ & & & 7.78 & 8.00 & 8.00 & 8.08 & 8.08 & 8.22 & \\
\hline $\begin{array}{c}\text { tercera } \\
\text { generación }\end{array}$ & & & & & 8.06 & 8.07 & 8.07 & 8.11 & 8.11 \\
\hline cuarta & & & & & & & & & \\
\hline generación & & & & & & & 8.08 & 8.13 & 8.13 \\
\hline $\begin{array}{c}\text { quinta } \\
\text { generación }\end{array}$ & & & & & & & & & 8.09 \\
\hline
\end{tabular}

Fuente: Dirección de Evaluación del Fideicomiso Educación Garantiza da, con datos de la Coordinación Ejecutiva de Prepa Sí, 2012. La escala de calificaciones va del 1 al 10. 
La gráfica 2, muestra la distribución de los ingresos de las familias de los beneficiarios del programa agrupados por categorías, donde el ingreso es medido en términos del número de salarios mínimos mensuales para el ciclo escolar 2011-2012.

\section{Gráfica 2}

Distribución de los ingresos de las familias de los beneficiarios del programa Prepa Sí, para el ciclo escolar 2011-2012

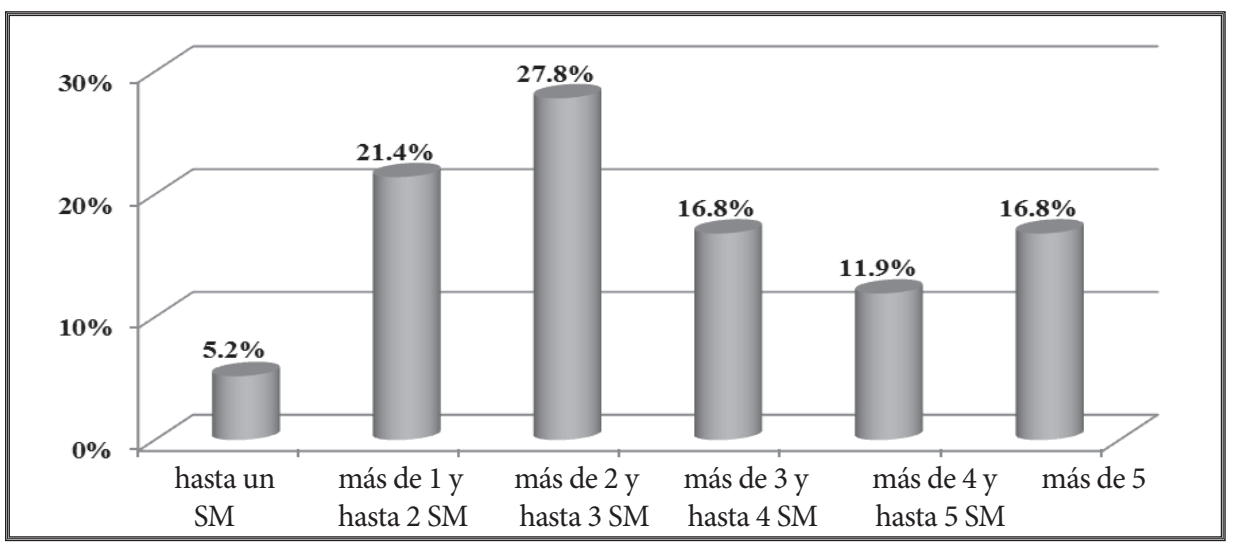

Fuente: Dirección de Evaluación del Fideicomiso Educación Garantizada, con información de la "Encuesta de Seguimiento a Beneficiarios del Programa Prepa Sí, ciclo escolar 2011-2012. Un salario mínimo en el año 2012, equivalía a 1500 pesos mensuales.

En cuanto a la opinión que tenían los beneficiarios acerca del programa, el 70\% de los entrevistados como parte de la encuesta de junio de 2012, indicaron que el estímulo es muy importante para que ellos puedan continuar sus estudios, dado que tienen problemas familiares, escolares y económicos. Por lo tanto, el estímulo les ayuda a mitigar esa situación. En promedio, los beneficiarios entrevistados de la muestra dieron al programa una calificación de 8.7 (en una escala de 1 a 10).

\subsection{Análisis econométrico de los determinantes del desempeño académico}

Dado que los determinantes del desempeño académico de los beneficiarios de Prepa Sí pueden ser de diferente índole (condiciones escolares, personales o socioeconómicas), para detectar los efectos del programa, se estimó un modelo 
econométrico a partir de la información proveniente de dos encuestas levantadas entre los beneficiarios del programa durante el ciclo escolar 2008-2009.

La ecuación 1 muestra el modelo econométrico semilogarítmico empleado, el cual permitió estimar el cambio relativo en el promedio de calificaciones de los beneficiarios del programa, en función de condiciones académicas, socioeconómicas y personales:

$$
\ln (\text { average })=\beta_{0}+\gamma Z+\mu
$$

Donde mide la variación relativa del promedio de calificaciones, debido a cambios absolutos en las variables de control (Z). Las variables utilizadas en el modelo para detectar el impacto del programa en el desempeño académico de los beneficiarios, fueron:

1) UNAM. Variable cualitativa que muestra si el beneficiario es estudiante la Universidad Nacional Autónoma de México, o de otra institución académica, bajo el supuesto de que la UNAM es una institución que capta a los mejores estudiantes del sistema público de educación media superior de la Ciudad de México, lo que hace que la mejoría en el desempeño académico de sus estudiantes dependa menos del estímulo que reciben del programa.

2) Género. Variable cualitativa que muestra el género del estudiante, donde 1 (uno) se refiere a las mujeres y 0 (cero) a los hombres. El supuesto aquí es que las mujeres en la sociedad mexicana se encuentran en desventaja frente a los hombres por cuestiones estructurales, de manera que ellas pueden desempeñarse mejor que los hombres cuando se les da la oportunidad de estudiar, que es lo que hace el estímulo de Prepa Sí.

3) Edad. Muestra la edad de los beneficiarios, medida en años. Se supone aquí que conforme el estudiante tiene mayor edad, menor es la presión a que mejore su desempeño.

4) Años de escolaridad de la madre. Variable que mide los años de escolaridad de la madre del beneficiario, bajo el supuesto de que las madres mejor educadas tradicionalmente les ayudan a sus hijos para que tengan un buen desempeño académico, de manera que es poco probable que se genere una mejoría en el desempeño de esos estudiantes, atribuible sólo al estímulo del programa.

5) Ingreso. Variable que muestra el ingreso mensual promedio del hogar del beneficiario, clasificado en una escala del 1 al 9, donde el valor de 1 se asigna 
a los hogares con los niveles más bajos de ingreso, y de 9, a los que tienen los niveles más altos. El supuesto aquí es que a mayor nivel de ingreso familiar, menor es la probabilidad de que el estudiante mejore su desempeño académico a causa del estímulo del programa, sobre la base de que el alcanzar un nivel alto de ingreso es un incentivo para mejorar el desempeño.

6) Trabaja. Variable que indica si el beneficiario tiene un trabajo por el que recibe una remuneración. Esta variable se incluye en el modelo para detectar la necesidad de trabajar del estudiante para obtener ingresos y ayudar a resolver las necesidades financieras en el hogar. Sin embargo, el trabajar disminuye el tiempo disponible para estudiar, y por tanto influye negativamente en el desempeño académico de los estudiantes, de manera que el estímulo puede contrarrestar ese efecto.

7) Situación de inscripción. Variable que muestra si el estudiante está inscrito como alumno regular (valor de 1) ó no (valor de 0 ). Se basa sobre el supuesto de que los estudiantes 'regulares' tienen mejores desempeños porque están más orientados a estudiar, de manera que el efecto del estímulo puede ser mayor en estos casos.

8) Ciclo Escolar 2009-2010.Variable que indica el ciclo escolar que cursa el beneficiario, donde el ciclo 2008-2009adopta el valor de 1, mientras que el ciclo 2007-2008 adopta el valor de 0 (cero). Ésta es sólo una variable de control.

La estimación de la ecuación 1 se realizó en dos etapas. En la primera etapa se aplicó el método de regresión de mínimos cuadros ordinarios (MCO) para estimar por separado la ecuación referente a los ciclos escolares 2007-2008 y 2008-2009. Los resultados de dichas estimaciones se muestran en el cuadro 3.

En una segunda etapa, se usó análisis de panel (combinación de corte transversal y series de tiempo), dado que las muestras para los ciclos 2007-2008 y 20082009 incluían a individuos diferentes (Wooldridge, 2002). Los datos del cuadro 3 , muestran que la edad es una variable muy influyente que afecta el desempeño académico de los estudiantes, de manera que por cada año adicional de edad, los beneficiarios obtienen un promedio de calificaciones más bajo, en $-0.03 \%$ en el ciclo escolar 2007-2008, y en -0.11\% para el ciclo 2008-2009. ${ }^{14}$

\footnotetext{
${ }^{14}$ Este resultado es importante ya que la edad aumenta los efectos de la variable del Ingreso en el aprovechamiento escolar. En estudios previos, los estudiantes reportaron (en un 31.3\%) que habían abandonado sus estudios por falta de ingresos, y por ello la tasa de deserción era más alta entre estudiantes de mayores edades. También se muestra que los estudiantes que provienen de hogares más pudientes, mostraban mejores niveles de desempeño académico.
} 


\section{Cuadro 3}

Modelo semilogarítmico para el análisis de los determinantes del desempeño académico de los beneficiarios del programa Prepa Sí

\begin{tabular}{|c|c|c|c|}
\hline \multirow[b]{2}{*}{$\begin{array}{l}\text { Variables } \\
\text { exógenas }\end{array}$} & \multicolumn{3}{|c|}{$\begin{array}{c}\text { Variable endógena: logaritmo natural del promedio } \\
\text { de calificaciones }\end{array}$} \\
\hline & $\begin{array}{c}\text { MCO } \\
\text { Muestra de primera } \\
\text { encuesta }\end{array}$ & $\begin{array}{c}\text { MCO } \\
\text { Muestra de segunda } \\
\text { encuesta }\end{array}$ & $\begin{array}{c}\text { Modelo panel } \\
\text { (corte transversal y } \\
\text { series de tiempo) }\end{array}$ \\
\hline \multirow[t]{2}{*}{ UNAM } & 0.009 & 0.039 & .065 \\
\hline & $(1.27)$ & $(5.26)^{* *}$ & $(5.96)^{* *}$ \\
\hline \multirow[t]{2}{*}{ Edad } & -0.0003 & -0.011 & .003 \\
\hline & $(0.07)$ & $(3.86)^{* *}$ & $(0.78)$ \\
\hline \multirow[t]{2}{*}{ Género } & 0.042 & 0.042 & .072 \\
\hline & $(4.67)^{* *}$ & $(6.13)^{* *}$ & $(2.92)^{*}$ \\
\hline \multirow[t]{2}{*}{ Ingreso } & 0.005 & 0.004 & -.006 \\
\hline & $(1.87)+$ & $(2.14)^{*}$ & $(0.84)$ \\
\hline \multirow[t]{2}{*}{$\begin{array}{l}\text { Escolaridad de } \\
\text { la madre }\end{array}$} & -0.002 & 0.001 & -.002 \\
\hline & (0.39) & -0.37 & $(0.48)$ \\
\hline \multirow[t]{2}{*}{$\begin{array}{l}\text { Situación de } \\
\text { inscripción }\end{array}$} & 0.132 & 0.092 & \\
\hline & $(10.86)^{* *}$ & $(8.42)^{* *}$ & \\
\hline \multirow[t]{2}{*}{ Trabaja } & -0.012 & 0.001 & -.023 \\
\hline & $(0.61)$ & (0.14) & $(0.75)$ \\
\hline \multirow[t]{2}{*}{$\begin{array}{l}\text { Ciclo escolar } \\
2009-2010\end{array}$} & & & .078 \\
\hline & & & $(17.63)^{*}$ \\
\hline $\begin{array}{l}\text { Número de } \\
\text { observaciones }\end{array}$ & 1950 & 1079 & 2195 \\
\hline$R^{2}$ & 0.31 & 0.36 & 0.237 \\
\hline
\end{tabular}

Nota: los valores absolutos de ' $z$ ' se incluyen entre paréntesis.

+ estadísticamente significativo al 10\%; ${ }^{* *}$ estadísticamente significativo al 1\%; ${ }^{*}$ estadísticamente significativo al 5\%.

Fuente: Dirección de Evaluación del Fideicomiso Educación Garantizada, con información de la primera y segunda encuestas de evaluación del programa Prepa Sí (2009). 
Otros resultados importantes incluyen los efectos positivos significativos que en el desempeño académico ejercen el Género, el Ingreso, la Situación de inscripción de los estudiantes, y la institución académica donde estudian (UNAM). El Género trabaja a favor de los estudiantes hombres y, como era de esperar, los estudiantes inscritos como alumnos regulares se desempeñan mejor que los irregulares. ${ }^{15}$ Los resultados también sugieren que los estudiantes de la UNAM se desempeñan mejor que los que asisten a otras instituciones públicas de educación superior en la ciudad, y que las familias con mayores ingresos tienen estudiantes que se desempeñan mejor.

\subsection{Análisis econométrico de los determinantes de la asistencia escolar}

Los determinantes de la deserción escolar han sido estudiados desde muy diversos enfoques, dado el gran número de factores que pueden estar involucrados por su alta complejidad. Tales factores podrían incluir tanto a las características personales de los estudiantes como otros factores involucrados en el proceso de integración de los estudiantes las instituciones sociales. ${ }^{16}$

Con la ayuda de un modelo econométrico probabilístico se intentó detectar los factores que influyen en la decisión de los beneficiarios del programa en cuanto a seguir o no asistiendo a la escuela. Específicamente, se trató de ver la influencia que el estímulo económico del programa afectaba esa decisión. ${ }^{17}$

El modelo se estimó con la información recabada mediante las encuestas de seguimiento levantadas entre los beneficiarios del programa, a partir de las respuestas a la pregunta si el estímulo les permitía continuar asistiendo a la escuela. El modelo estimado se muestra en la ecuación 2:

\footnotetext{
${ }^{15}$ Un resultado interesante, no esperado, fue el efecto estadísticamente no-significativo en el desempeño académico derivado del hecho de que los estudiantes tuviera un empleo. Se podría esperar que trabajar les resta tiempo para estudiar, pero ése no fue el caso entre los beneficiarios de la muestra.

${ }^{16}$ En campos del conocimiento como la sociología o la sicología, los investigadores se han enfocado en la personalidad de los estudiantes, tanto como en otros factores (externos a los estudiantes) que caracterizan sus relaciones sociales. Algunos de ellos han argumentado que junto con las condiciones económicas de sus familias, esos factores sociales contribuyen a la preservación de las desigualdades socioeconómicas dentro de la sociedad en países en desarrollo (Ebrard et al., 2009).

${ }^{17}$ El modelo econométrico utilizado para el ejercicio fue un modelo Probit binario, donde $P\left(y_{i}=1\right)$ indica la probabilidad de que el estudiante permanezca en la escuela gracias a la ayuda económica derivada de Prepa Sí, y es una función $F$, de un conjunto de variables $(X)$ que incluyen información sobre las condiciones de inscripción de los estudiantes, así como de otras variables socioeconómicas explicativas (Z), tales como género, edad, años de escolaridad de la madre, promedio de calificaciones del estudiante, o condiciones laborales de los estudiantes, entre otras.
} 


$$
P\left(y_{i}=1 \mid X\right)=F\left(\beta_{0}+\beta_{1} X+\gamma Z\right)
$$

Donde $P\left(y_{i}=1\right)$ muestra la probabilidad de que el beneficiario siga asistiendo a la escuela gracias al estímulo recibido; $F$, indica la función que permite que los valores de $P$ se encuentren entre 0 (cero) y 1 (uno); $X$ contiene la información sobre las condiciones de inscripción del estudiante, $y$, $Z$ es un vector de variables socioeconómicas explicativas.

Las variables consideradas para la estimación del modelo fueron las siguientes:

1) Ingreso. Variable que indica el ingreso promedio mensual del hogar del beneficiario, en una escala de 1 a 9, donde el valor de 1 se asigna a los hogares con los valores más bajos de ingreso, y el 9 corresponde a quienes tienen los más altos. El supuesto aquí es que conforme el ingreso es mayor, menor es la probabilidad de que el estudiante abandone la escuela, de manera que el estímulo económico es más importante para los estudiantes provenientes de hogares con ingresos bajos, aunque puede darse la situación de que el estímulo refuerce la decisión de permanecer en la escuela en todos los casos.

2) Índice de bienestar material familiar (IBMF). Índice que muestra la suma de los implementos y condiciones de la vivienda familiar (materiales de construcción de la vivienda, electrodomésticos disponibles, entre otros), de manera que a mayor valor del índice, mejores serían también las condiciones de bienestar material de la familia. Los rangos establecidos para dicho índice fueron: $a$ ) muy bajo $(0-1)$; $b$ ) bajo (2-3); intermedio (4-6), y; $d$ ) alto (7-9). El supuesto aquí es que a valores más altos del índice, menos importante es el estímulo del programa como determinante de que el estudiante permanezca en la escuela.

3) Género. Variable cualitativa que indica el género de la beneficiaria (valor de 1) ó del beneficiario (valor de 0). Aquí el supuesto es que las mujeres en México tienen condiciones de desventaja con respecto a los hombres, por razones estructurales, de manera que ellas aprovechan mejor la oportunidad que les otorga el programa de asistir a la escuela, gracias al estímulo.

4) Edad. Variable que muestra la edad de los beneficiarios, medida en años. Se supone aquí que mientras mayor edad tengan los beneficiarios, mayor es la presión que tienen para abandonar la escuela, por efectos laborales o de responsabilidad familiar, y menor la importancia 
del estímulo en la retención.

5) Estado civil. Variable que muestra el estado civil de los beneficiarios, donde 1 (uno) significa que el beneficiario es soltero, y 0 (cero) significa un estado civil diferente. El supuesto en este caso es que los estudiantes casados tienden a abandonar la escuela más fácilmente que los solteros, dada la presión de atender a sus familias, de manera que el estímulo puede ser decisivo para que los estudiantes casados permanezcan en la escuela.

6) Años de escolaridad de la madre. Variable que indica los años de escolaridad de la madre del beneficiario, sobre el supuesto de que las madres mejor educadas le asignan un valor muy alto al hecho de que los hijos asistan a la escuela, por lo que aprecian mucho cualquier ayuda que les permita asegurarse de continuar asistiendo.

7) Trabaja. Variable que indica si el beneficiario tiene un empleo por el cual recibe alguna remuneración. Esta variable se introdujo con el objeto de detectar si el beneficiario tiene necesidad de trabajar para obtener ingresos que le permitan contribuir a las finanzas familiares, aumentando así la probabilidad de abandonar la escuela, pero también la importancia del estímulo.

8) Promedio. Variable que indica el promedio general de calificaciones que tenía el estudiante al momento de la encuesta. Aquí se supone que calificaciones altas son un incentivo fuerte para continuar en la escuela, y el estímulo puede reforzar ese incentivo.

Los resultados de la estimación de la ecuación 2 se muestran en el cuadro 4. Dichos resultados sugieren que las variables que reciben la mayor influencia del estímulo en la decisión de permanecer en la escuela por parte de los estudiantes son el Ingreso y el Índice de bienestar material familar. En el caso del Ingreso, la probabilidad de que el estímulo económico del programa influya en la decisión de no abandonar la escuela es más alta entre estudiantes de familias con niveles bajos de ingresos, lo que sugiere que la falta de ingreso es un factor que puede obligar a los estudiantes a abandonar la escuela.

Resultados semejantes se obtuvieron con el IBMF, en el sentido que a valores más bajos del índice, más fuerte es el efecto del estímulo económico en la decisión de continuar estudiando, por parte del beneficiario.

Los otros dos resultados estadísticamente significativos se refieren al Género, y a los Años de escolaridad de la madre. Esos resultados indican que los hombres muestran una probabilidad menor (de menos 5\%) que las mujeres en cuanto a continuar estudiando gracias al estímulo económico. Los resultados 
también muestran que los estudiantes que provienen de familias con menores niveles de educación (años de escolaridad) dependen del estímulo para seguir estudiando, en grados mayores a los que provienen de familias más educadas. ${ }^{18}$

\section{Cuadro 4}

Modelo econométrico para estimar los efectos del estímulo económico del programa Prepa Sí en la prevención del abandono escolar

\begin{tabular}{|lc|}
\hline \multicolumn{1}{|c|}{ Variables exógenas } & $\begin{array}{c}\text { Variable endógena: } \\
\text { "el estímulo me permite } \\
\text { continuar estudiando" }\end{array}$ \\
\hline \hline Edad & -0.007 \\
Género & $(0.93)$ \\
Estado civil (casado) & -0.05 \\
Ingreso & $(1.86)+$ \\
IBMF & -0.052 \\
& $(0.58)$ \\
Años de escolaridad de la madre & -0.040 \\
Promedio & $(5.19)^{* *}$ \\
Trabaja & -0.030 \\
Número de observaciones & $(2.04)^{*}$ \\
Pseudo ${ }^{2}$ & -0.021 \\
\hline
\end{tabular}

Nota: valores absolutos de " $z$ " se incluyen entre paréntesis.

+ estadísticamente significativo al 10\%; ${ }^{* *}$ estadísticamente significativo al 1\%; ${ }^{*}$ estadísticamente significativo al 5\%.

Fuente: Dirección de Evaluación of Fideicomiso Educación Garantizada, con información de la primera y segunda encuestas de seguimiento del programa Prepa Sí (2009).

${ }^{18}$ Los resultados sugieren que las otras variables consideradas, aunque mostraron los signos esperados, no fueron lo suficientemente fuertes o estadísticamente significativos como para explicar la influencia del estímulo económico asociado con Prepa Sí, en la retención escolar. 
Tales resultados indican que Prepa Sí cumple correctamente con su objetivo principal de evitar que los estudiantes abandonen la escuela por motivos económicos, ya que el estímulo es recibido por grandes proporciones de estudiantes que provienen de familias con niveles bajos de educación, con fuertes dificultades económicas, y con bajos niveles de bienestar material.

\section{Consideraciones finales y conclusiones}

Como se mencionó en la introducción, el objetivo principal en este trabajo es presentar los resultados de una evaluación de los impactos de Prepa Sí en sus objetivos asignados, que incluyen el abatimiento de la tasa de deserción y el mejoramiento del desempeño escolar de los beneficiarios de este programa, realizada recientemente por el autor, con la ayuda del análisis econométrico, y siguiendo los lineamientos de la metodología utilizada comúnmente en los casos de programas universales.

Después del análisis de las características del programa, que puede considerarse como de carácter universal, ya que está dirigido a todos los estudiantes de bachillerato inscritos en escuelas públicas localizadas en la ciudad de México, se puede concluir que Prepa Sí fue diseñado e implantado sobre el reconocimiento de derechos sociales por parte del gobierno de la ciudad, y con la aceptación de la responsabilidad de cumplirlos y hacerlos cumplir, y se ajusta a una combinación de los modelos residual e institucional-redistributivo propuestos por Montoro (1997), dado que el programa intenta asegurar que los servicios sociales y educativos que ni la familia ni el mercado pueden proveer, lleguen a los estudiantes, y éstos puedan tener la oportunidad de aprovechar los avances en la educación.

Pero Prepa Sí reúne también las características del modelo normativo en la clasificación de Montoro (1997), de acuerdo con el cual se evalúan los programas en términos de la discrepancia entre su diseño y los resultados.

Finalmente, los resultados del análisis econométrico de los determinantes de la retención y el aprovechamiento escolar de los estudiantes, sugieren que el programa ha tenido los resultados que se esperaban de él, y que sus instrumentos principales, incluidos el estímulo económico asociado con el mismo y las actividades en comunidad que realizan sus beneficiarios, se encuentran entre las variables más efectivas a considerar al tratar de resolver el problema de la deserción escolar en el nivel medio superior en la Ciudad de México, e incluso tal vez en todas las regiones menos desarrolladas. 


\section{Referencias}

Baker, J. (2000). Evaluating the Impact of Development Projects on Poverty. A Handbook for Practitioners. Washington, D.C., The World Bank.

Donati, P.P. (1985). Le Frontiere della Politica Sociale. Milán: Franco Angeli.

Ebrard-Casaubon, M. L.; M. M. Carrillo-Huerta y J. A. Cerón-Vargas (2009). La política de desarrollo a favor de la educación: el caso del programa Prepa Sí de la Ciudad de México. México, DF: Subsecretaría de Educación Media Superior y Superior del Gobierno del Distrito Federal, Universidad de Puebla y Galilei.

Fideicomiso Educación Garantizada (2008). Primera Encuesta de Evaluación del Programa de Estímulos para el Bachillerato Universal (PREBU, Prepa Sí) 20072008. México, DF, Dirección de Evaluación del FIDEGAR.

Heckman, J. (1979). "Sample Selection as a Specification Error". Econometrica, núm. 47, pp.153-161.

(2001). "Micro Data, Heterogeneity, and the Evaluation of Public Policy". Journal of Political Economy, 109, pp. 673-748.

Heckman, J.; H. Ichimura, J. Smith y O. Todd (1998). "Characterizing Selection Bias Using Experimental Data”. Econometrica, núm. 66, pp. 1017-1098.

Hernández-Licona, G. (2004). Las políticas públicas de la evaluación de programas sociales. México, DF: SEDESOL.

Higgins, H. (1980). "Social Control Theories of Social Policy". Journal of Social Policy, vol. 9, núm. 1, pp. 76-102.

INEGI. (2006). Censo de Población y Vivienda 2005; Estadísticas Básicas del Sistema Educativo Nacional, Fin de Cursos 2005-2006. México, DF, INEGI, Disponible en http://www.inegi.org.mx. (2010). II Censo General de Población y Vivienda 2010. México, DF: Instituto Nacional de Geografía y Estadística.

Instituto Mexicano de la Juventud. (2006). Encuesta Nacional de Juventud 2005. México, DF, INJUVE, disponible en: http://imjuventud.gob.mxhttp://urbared. sociales.unam.mx/textos/encuesta_juventud_2005.pdf.

Mishra, R. (1990). El Estado de Bienestar en la sociedad capitalista. Madrid: Ministerio de Asuntos Sociales.

Montoro-Romero, Ricardo (1997). "Fundamentos teóricos de la política social", en Alemán, C. y J. Garcés (Coordinadores). Política social. Madrid: McGraw-Hill, pp. 33-50. 
Subsecretaría de Educación Media Superior y Superior del Distrito Federal (2008). Encuesta: Nuestras Actividades Comunitarias. Dirección de Evaluación de la SEMM del Distrito Federal. México, DF.

Wooldrige, J. (2002). Econometric Analysis of Cross Section and Panel Data. Cambridge, Mass., MIT Press. 